\title{
Convalescent plasma as therapeutic agent to treat Ebola
}

\section{Introduction}

During the West African Ebola outbreak in 2014-2015 several affected countries prioritized the use of convalescent plasma obtained from Ebola patients to treat persons infected with the disease. While some Ebola patient actually got cured as a result of convalescent Ebola plasma transfusion yet many also died. Several studies were conducted to assess the efficacy of using convalescent Ebola plasma to treat Ebola patients though with conflicting results. In one nonrandomized study of 99 confirmed Ebola patients with different demographics who received two consecutive 200-250 $\mathrm{ml}$ of ABOcompatible convalescent plasma transfusions, the risk of death in the convalescent-plasma group was lower (31\%) than that for the control group (38\%) with a $95 \%$ confidence interval of -18 to 4 .

The use of convalescent Ebola plasma was a contentious issue during the West African Ebola outbreak in 2014-2015. In addition to the risky and complex nature of generally treating and managing Ebola cases the therapeutic use of convalescent Ebola plasma is also fraught with ethical issues which will also have long term financial complications.

First, because pharmaceutical companies are known for raking billions of dollars in selling their products, Ebola survivors whose convalescent Ebola plasma is used as therapeutic agent can impose huge financial strain on these biotech companies for using their biological products to the point that the companies may not be able to use it or forced to use it but at an increased cost at the expense of the patient. This huge cost can additionally deter such biotech companies, big and small, from innovating and researching in the further use of convalescent Ebola plasma as a therapeutic agent which will invariably impact negatively on those developing African countries that mostly experience Ebola outbreaks.

Secondly, although the American Food and Drugs Administration (FDA) and other drug evaluation and monitoring agencies have been making efforts to minimize drug development costs and have been incentizing pharma to develop less-profitable drugs, it is widely believed that these steps may have limited and less significant financial impact on the case of Ebola that is mostly associated to Africa countries. The periodic nature of Ebola outbreaks makes it a less interesting area for FDA involvement and incentization.

One way to go round such potential bottle necks surrounding the use of convalescent Ebola plasma to treat future Ebola patients is to get these pharm companies to build high tech laboratories in these Ebola-prone regions that will serve as standby Ebola compassionate treatment units in the event of an outbreak. This is justified because it will be unwise for biotech companies with interest in using convalescent Ebola plasma for therapeutic use to be located far away from the epidemic cycle given the contagious nature of Ebola. Also considering the fact that the designing of a therapeutic agent for Ebola is quite different from other infectious agents who typical involve testing the trial drug in fewer than 1000 s of healthy volunteers without the risk of an outbreak, designing a therapeutic agent for Ebola increases the risk of an Ebola outbreak. Although the different
Volume I Issue I - 2017

Jia B Kangbai
PhD medical research fellow, Ludwig Maximilians University,
Germany

Correspondence: Jia B Kangbai, PhD medical research fellow, Centre for International Health, Ludwig Maximilians University in Munich Germany, Tel 232-7865-5460, Email jiakangbai@hotmail.com

Received: April 0I, 2017 | Published: April 10, 2017

phases of clinical trials have varying financial cost yet the cost of a typical Phase 3 randomized control trials for a new drug is generally high since it involves the recruitment of hundreds or thousands of patients mostly across many countries and require lots of laboratory time to assess the new drug efficacy. The periodic outbreak of Ebola alongside its high case fatality rates makes the aggregation of large number of Ebola cases for such clinical trials almost impossible to attain.

Finally, the various biotech companies and pharmaceuticals interested in using convalescent Ebola plasma should start exploring ways to create or partner with nations such as Uganda, Democratic Republic of Congo, Sierra Leone, Liberia and Guinea that have experience large scale Ebola outbreaks in the past on how they can work out strategies for the large scale use of convalescent Ebola plasma to treat future Ebola cases. Their strategies should not be devoid of future Ebola survivors whose convalescent Ebola plasma will be used after all. In Sierra Leone most Ebola survivors whose convalescent Ebola plasma were used to treat Ebola infected patients during the 2014-2015 West African Ebola outbreak were suspicious about the use of their biomedical product. These convalescent Ebola plasma donors often claim being manipulated by big pharm companies and local authorities for the release of their convalescent Ebola plasma although on compassionate grounds.

Agreed that drug companies have been provided reliable methods to bring new products to the market the scope and scale of the disease under consideration makes existing drug marketing methods less workable. As expensive pharmaceuticals tries to introduce new drug therapies including convalescent Ebola plasma drug control and evaluation, these companies should consider creative and innovative ways to reduce the cost of their product without harming the safety of patients or engage in unethical means to achieve their objectives.

\section{Acknowledgements}

None.

\section{Conflict of interest}

The author declares no conflict of interest. 